\title{
Geospatial analysis of environmental risk factors for missing dementia patients
}

Vaisakh Puthusseryppady ${ }^{1}$, Gillian Coughlan ${ }^{1}$, Martyn Patel $^{1,2}$, Michael Hornberger ${ }^{1,3 *}$

${ }^{1}$ Norwich Medical School, University of East Anglia, Norwich NR4 7TJ, UK.

${ }^{2}$ Norfolk and Norwich University Hospitals NHS Foundation Trust, Colney Lane, Norwich NR4 7UY, UK

${ }^{3}$ Dementia and Complexity in Later Life, NHS Norfolk and Suffolk Foundation Trust, UK

Corresponding author: Prof. Michael Hornberger, Norwich Medical School, 2.04 Bob

Champion Research and Education Building, University of East Anglia, Norwich NR4 7TJ, UK; phone: 0044 (0) 160359 7139; email: m.hornberger@uea.ac.uk.

Running Title: Geospatial analysis of missing incidents

Words: 4,079 


\begin{abstract}
BACKGROUND: Dementia-related missing incidents are highly prevalent but still poorly understood. This is particularly true for environmental/geospatial risk factors, which might contribute to these missing incidents.
\end{abstract}

OBJECTIVE: The study aimed to conduct a retrospective, observational analysis on a large sample of missing dementia patient case records provided by the police $(n=210)$, covering dates from January 2014 to December 2017. In particular, we wanted to explore i) hotspot regions of missing incidents and ii) the relationship between outdoor landmark density and missing incidents.

METHODS: Global spatial autocorrelation (Moran's I) was used to identify the potential hotspot regions for missing incidents. Meanwhile, spatial buffer and regression modelling were used to determine the relationship between landmark density and missing incidents.

RESULTS: Our demographics measures replicated and extended previous studies of dementia-related missing incidents. Meanwhile, no hotspot regions for missing incidents were identified, whilst higher outdoor landmark density lead to increased missing incidents.

CONCLUSION: Our results highlight that missing incidents do not occur in isolated hotspots of regions but instead are endemic in patients regardless of location. Higher landmark density emerges as a significant geospatial factor for missing incidents in dementia, which crucially informs future safeguarding/intervention studies.

Keywords: Dementia, Spatial navigation, Spatial analysis, Risk factors 


\section{Introduction}

A dementia-related missing incident is defined as an instance where a patient is not at an expected location, and when their whereabouts are unknown to the carer [4]. Up to $70 \%$ of dementia patients experience at least one missing incident, with an estimated 40,000 patients going missing for the first time in the UK every year [5]. Moreover, some patients are also at risk for getting lost multiple times [6,7]. These missing incidents most commonly occur when the dementia patient is temporarily unsupervised, such as when they perform a routine activity (eg. Walking in the neighbourhood) or even during the night when the carer is sleeping $[8,9]$.

Dementia-related missing incidents have been suggested to be a symptom that is more prominently seen in Alzheimer's Disease (AD) as opposed to other dementia types [1,2], and stem from fundamental deficits to the spatial navigational abilities caused by the AD neuropathology in patients. In particular, pathology induced alterations to the medial temporal and parietal lobe structures result in impairments to the two main navigation strategies - egocentric (body-based) and allocentric (map-based) navigation respectively, as well as the interaction between the two [3]. Indeed, these impairments play a key role in contributing to patients feeling spatially disoriented and as such, getting lost in the community. The occurrence of missing incidents negatively impacts patients by increasing their chances of institutionalization, reducing their sense of autonomy, and in worst case scenarios lead to harm or death $[7,10]$. It also significantly increases patients' carers/families stress and triggers the increasing involvement of law enforcement (i.e., police) as well as community search resources $[9,11-13]$.

Despite missing incidents being such a prevalent problem, the exact nature and category of the most important factors contributing to these incidents are still poorly understood. To date, most studies have focused on contextual, situational, and neurocognitive factors contributing 
to missing incidents in dementia [8], with virtually no studies investigating how real-life environmental factors might influence these incidents. With the environment playing an integral role in real-world navigation, identifying such factors is of clear importance for not only the prediction of these incidents but also for improving the safeguarding of patients to prevent these incidents from occurring again in the future.

In the current study, we investigate potential environmental factors that might contribute to missing incidents in dementia. We employ geospatial analytical techniques which are increasingly used in health and disease studies[14], to investigate the spatial patterns of dementia-related missing incidents. In particular, we investigate whether there are any locations that exhibit higher risk for patients to go missing from (i.e., hotspots). Moreover, with landmarks being important entities that are used for real world navigation, we also investigate whether landmark density might be a contributing factor to missing incidents. To this purpose, we conducted a retrospective analysis of police records of missing incidents of dementia patients over a 3-year period. We predicted that there would be no 'hotspots' for missing incidents, once controlling for population density, as spatial disorientation is now seen as an integral part of dementia [3]. By contrast, we predicted that lower landmark density would lead to higher incidence of missing incidents, as patients will not be able to use distinct landmarks for navigating safely back to their starting point.

\section{Methods}

\subsection{Study Design}

Records of missing patients with dementia (MPWD) were provided by the Norfolk police force with a total of 210 anonymised cases covering dates from January 2014 to December 2017, for the Norfolk county (total population 898,390) in the United Kingdom (UK). 
For each missing case, the following data was provided - date missing, gender, age, location missing from (town and postcode), type of setting missing from (care home/hospital, domestic residence, public), location found (building name/road and town), case details (circumstances in which patient went missing/was found), time missing (minutes), and whether it was the first time missing (yes/no). From the location missing from/found information, the distance travelled by each MPWD was calculated in OpenStreetMap by using the shortest routes linking the two locations, which was determined by the mapping platform. Meanwhile, the locations patients went missing from were classified as urban or rural using the UK Office for National Statistic's 2011 rural urban classification guide [15]. Lastly, from the case details, we inferred whether the MPWD sustained harm (i.e., injuries/death) during the missing incident.

Ethical approval for this study was granted by the Faculty of Medicine and Health Sciences Research Ethics Committee at the University of East Anglia (Ref. 2017/18 - 94). 2.2 Data Analysis

\subsubsection{Demographics Analysis}

The MPWD data comprised of a mixture of continuous and categorical variables. ShapiroWilk normality tests were conducted on the continuous variables (age, time missing, and distance travelled) to determine whether to use parametric or non-parametric statistics tests on the data. Meanwhile, Chi-Square and where appropriate, Fisher's Exact Test were used to explore associations between the remaining, categorical variables. All demographics analysis were conducted via R software package version 3.4.2 [16]. 


\subsubsection{Global Spatial Autocorrelation Analysis: Hotspots for Missing Patients with Dementia}

Identification of spatial hotspots for the MPWD were conducted on ArcGIS software version 10.3.1 [17] with a map of the Norfolk County in the British National Grid projected coordinate system. The Norfolk county was sub-divided into its lower layer super output areas (LSOA) to provide specific spatial units for the analysis. LSOAs were chosen as they represent geographic units commonly used by the UK Office for National Statistics for reporting small area statistics (eg. neighbourhood population, income estimates, housing etc.) [18], and hence have good ecological validity by allowing to split the data into three main localities (urban, rural town and rural villages). For this analysis, we downloaded a shape file containing the UK sub-divided into its different LSOAs from the UK Office for National Statistics Open Geography Portal [19], and extracted only the LSOAs for the Norfolk region. In this shapefile, each LSOA was classified as being either urban or rural based on population density, and the latter were further sub-classified into rural towns and rural villages based on household density[15].

The locations patients went missing from were then plotted onto a map of Norfolk. In total, the 210 MPWD went missing from 168 different locations across the region (Fig. 1(i)), with there being 17 locations where multiple patients got lost. For patients that went missing multiple times, only one location (i.e., that of the most recent missing incident) was reported. In addition, there were 3 cases where the location the patient went missing from was not reported. All 168 missing patient locations were aggregated into the respective LSOAs in which they fell in. Of these 168 locations, 96 fell within urban LSOAs, 33 in rural town LSOAs, and 39 in rural village LSOAs. To control for different population densities across Norfolk, the number of MPWD falling within each LSOA was divided by the total population of that LSOA and multiplied by 10,000 to get a whole number. 
To identify the spatial hotspots for MPWD, a widely used geospatial analytical method known as global spatial autocorrelation (Moran's I) was used, which identifies potential spatial patterns evident across a region. This analysis explores the distribution of the normalised MPWD numbers across all LSOAs and tries to identify if the dataset exhibits spatial clustering (i.e., similar values occurring near each other) [20]. In this analytical approach, each LSOA is grouped together with its neighbouring LSOA's, forming what is termed as a neighbourhood. Following standard practice in geospatial analysis, the K-nearest (i.e., solution $=8$ ) neighbours approach was used to determine the neighbourhood for each LSOA unit, owing to the non-normal distribution of MPWD values across all LSOAs. This means that each LSOA along with its nearest 8 neighbours comprised a neighbourhood. The MPWD values in each respective neighbourhood across the region were then analysed to identify whether spatial clustering of similar values occurred in the dataset.

Any spatial dataset can exhibit one of three types of global spatial autocorrelation - positive (maximum value +1 ; clustering of values), zero (value of 0 ; completely random spatial pattern of values), or negative (maximum value of -1 ; spatial pattern where dissimilar values appear near each other) (Fig. 1(iii)). In theory, if the dataset exhibits either positive or negative global spatial autocorrelation, a follow up local spatial autocorrelation (Anselin Moran's I) would have to be run [21]. In the case of the former, the follow up analyses would reveal the spatial locations and extents of the clusters as well as whether these clusters are significant hotspots (i.e., exhibit relatively higher values compared to rest of region) or coldspots (i.e., exhibit relatively lower values compared to rest of region).

Typically, global spatial autocorrelation cannot be performed if there are spatial units exhibiting null values of a variable and consequently, all LSOAs not exhibiting MPWD locations were removed from the analysis region. A global spatial autocorrelation (Moran's I) analysis was then run on the remaining LSOAs (Fig. 1(ii)). 
(Insert Figure 1)

\subsubsection{Spatial Buffer \& Regression Analysis - Relationship of Outdoor Landmarks to Missing}

\section{Patients with Dementia}

A spatial buffer analysis was conducted to explore the relationship between the presence of outdoor landmarks and MPWD. This approach involves generating a buffer zone of a specific radius around each MPWD location and identifying the number of outdoor landmarks falling within these buffer zones. In essence, this enables us to estimate the outdoor landmarks that each MPWD would have encountered at the time and place they were reported missing. To run this analysis, a dataset containing all the landmarks in the Norfolk region, in shape-file format, was downloaded from OpenStreetMap and imported into ArcGIS. This dataset contained any object or location that fell into the following five categories - Amenity \& Leisure, Tourism, Traffic \& Transport, Urban \& Rural Furniture, and Historic (see supplementary material for full breakdown of landmark categories, subcategories, and tags). For each landmark, details of its name (e.g., Riverside Leisure Centre), type (Swimming Pool), and map co-ordinates (X,Y; in the World Geodetic System 1984 geographic coordinate system) were provided in the dataset. The landmarks in the shape-file were overlaid onto a map of the Norfolk LSOAs. Both maps utilised the World Geodetic System 1984 geographic co-ordinate system.

First, we searched and removed landmark duplicates in the dataset. Next, landmarks that fell inside other landmarks were identified and their visibility from open street view was examined using Google Maps. If such landmarks were not visible from street view (e.g. individual shops falling inside a shopping mall), they were removed from the dataset, as it is unlikely that the MPWD would have used or been exposed to this landmark whilst navigating. Meanwhile, landmarks falling inside other landmarks that were visible from street 
view were examined to see if they were at least as salient as the landmark they fell within, using Google Maps. If this condition was satisfied, (e.g., bell tower as part of a church), then these landmarks were kept in the dataset, as it may have been just as likely for either of these landmarks to have caught the attention of the MPWD whilst navigating. If the saliency condition was not satisfied, then those landmarks were removed. After controlling for all factors listed above, we ended up with a total of 24,900 outdoor landmarks for analysis.

Next, for each of the 168 MPWD locations, a geodesic buffer zone with a radius of 1 kilometre was generated and the number of outdoor landmarks falling within each buffer zone was computed (Fig. 2(i), (iii)). Following this, a set of 168 random, control locations were generated across the entire Norfolk region using an in-built algorithm in ArcGIS (Fig. 2(ii)). These random locations were generated in regions falling outside the MPWD location buffer zones, and controlled to have the same urban/rural distribution as the MPWD locations (96 urban locations, 33 in rural town, 39 in rural villages). The random locations were also controlled for the type of land they fell in. Of the 96 urban MPWD locations, 2 fell in industrial \& retail lands, 69 in residential lands, and 23 in unclassified lands. Of the 33 rural town MPWD locations, 1 fell in forest lands, 25 in residential lands, 2 in retail lands, and the remaining 5 in unclassified lands. Lastly, of the 39 rural village MPWD locations, 29 fell in residential lands, 1 in commercial lands, and 9 in unclassified lands. The same number of random location points for each land use type were generated across Norfolk, for each respective locality (urban, rural town, rural village).

Once all 168 random locations were generated, geodesic buffer zones with a 1 kilometre radius were generated for each location and the number of outdoor landmarks falling within each location's buffer zone was computed (Fig. 2(iv)). As the number of outdoor landmarks in both the MPWD locations and random locations had a non-normal distribution, a 
Wilcoxon Rank Sum test was run to compare the number of outdoor landmarks falling within the buffer zones of both groups.

\section{(Insert Figure 2)}

To explore the relationship between presence of outdoor landmarks and MPWD, on a more global scale, ordinarily least squares regression models were run where the number of MPWD in each LSOA were respectively regressed against the landmark density for each LSOA. In total, three independent regression models were run - one for urban, rural town, and rural village regions respectively. All regression models were run in R software.

\section{Results}

\subsection{Demographics Risk Factors}

The MPWD demographics (Table 1) showed similar numbers of MPWD getting lost across all 4 seasons, as well as similar numbers of males and females getting lost. The majority of MPWDs went missing from domestic residence settings $(n=134)$ when compared to care facilities $(n=52)$ or general public locations $(n=23)$. A total of 86 MPWD went missing on foot, 33 cases used some form of transport (taxi/bus/train/car), 2 cases used a combination of transport and foot, and the remaining 87 cases did not have sufficient information provided to infer their mode of transportation. Subgroups of MPWD that went missing multiple times ( $n$ $=52)$, as well as those that sustained harm whilst lost $(n=10)$, were also identified. All MPWD were found alive except for one case.

(Insert Table 1) 
There is often a general assumption that spatial navigation might be different between male and females $[22,23]$. We therefore explored associations between the getting lost variables and sex as a factor. For the type of location MPWD went missing from, the results showed that MPWD missing from domestic residences were more likely to be female than male $\left(\mathrm{X}^{2}=8.644524, \mathrm{p}=0.013\right)$. By contrast, MPWD who go missing multiple times were more likely to be male than female $\left(X^{2}=7.701392, p=0.005\right)$. Lastly, the results showed that male MPWD went missing for significantly longer periods than females $(\mathrm{W}=4293, \mathrm{p}=0.007)$.

Finally, we explored potential demographic risk factors for patients who went missing multiple times as well as for those who sustained harm. When comparing the patients missing multiple times to those that went missing only once, no significant differences were seen in any variable. However, a statistical trend was observed for age, with patients missing multiple times being younger than patients that only went missing once $(\mathrm{W}=4804, \mathrm{p}=$ 0.056). A statistical trend was also observed for distance travelled, with patients missing multiple times travelling a lower distance than those that only went missing once $(\mathrm{W}=$ $3766.5, \mathrm{p}=0.058)$. Meanwhile, no significant differences were observed in any variable when comparing patients which sustained harm to the unharmed patients.

\subsection{Spatial Hotspots Analysis}

The global spatial autocorrelation (Moran's I) analysis revealed no significant spatial autocorrelation in our dataset (Global Moran's $\mathrm{I}=-0.011675, \mathrm{p}=0.911)$. Considering that the global trend can potentially mask subtle underlying cluster like patterns present in specific regions, a follow up local spatial autocorrelation (Anselin Local Moran's I) analysis was run to identify possible underlying clusters. Here, a False Discovery Rate was used to correct for multiple comparisons. The results of the follow-up analysis confirmed the global spatial autocorrelation results, signifying that the MPWD exhibits a random spatial pattern 
across Norfolk and as such, there are no significant hotspots (or cold spots) for MPWD in the examined region.

\subsection{Spatial Buffer \& Regression Analysis}

The Wilcoxon Rank Sum Test revealed that there is a significantly higher number of outdoor landmarks falling within a 1 kilometre buffer zone of the MPWD locations when compared to the random locations $(\mathrm{W}=21312, \mathrm{p}<0.001)$. Moreover, the regression modelling showed a significantly positive relationship between outdoor landmark density and MPWD in urban as well as rural village regions ( $\mathrm{p}<0.001$ for both). However, no significant relationship was found in rural town regions $(\mathrm{p}=0.770)$. 


\section{Discussion}

As hypothesised, the spatial autocorrelation analysis revealed no hotspot locations for MPWD (i.e, MPWD are not more prone to get lost in certain regions compared to others). However, contrary to our hypothesis we found that increased landmark density was associated with increased missing incidents in patients.

On a demographic level, we replicated previous findings showing that the majority of MPWD went missing from domestic residences as opposed to care facilities [4,24,25], which is likely due to differences in the level of safeguarding mechanisms available in home vs. care locations. Moreover, it is likely that the MPWD living in domestic residences have greater opportunities to get outdoors than those that are institutionalised, thereby placing themselves in situations where they might be at risk of getting lost. Taken together, this suggests that missing incidents are a significantly greater problem for patients living at home compared to those in care facilities, even after controlling for the differences in ratio of dementia patients living in these residences (home - 61\%; care facilities - 39\%) in the UK [26]. This is indeed a factor that should be made aware to the police/rescue services and one that should be considered by health and social care professionals when developing care plans for patients residing at home. Despite this however, it should be mentioned that the number of missing incidents reported in each residence type should ideally also be controlled for the number of times the patients leave the premises in order to truly determine whether missing incidents are a greater problem in one type of residence over another.

Other demographics results revealed comparable numbers of patients getting lost across all 4 seasons as well as a similar number of male and female patients getting lost. The latter finding replicates previous findings $[24,25]$. We also found that male MPWD went missing for significantly longer and were associated with being more likely to go missing multiple times when compared to females. Moreover, in relation to multiple missing incidents we 
observed a statistical trend for younger age being associated with missing multiple times, consistent with a previous study [27]. Finally, we also found a very small group of MPWD who sustained harm whilst lost, however this was not related to any other demographic risk factors.

The global spatial autocorrelation analysis investigated the spatial distribution of where MPWD get lost (i.e. hotspots for missing incidents). Spatial autocorrelation analyses have so far only been used to establish the frequency and mortality of dementia across regions $[28,29]$, but to our knowledge, this is the first study to use this geospatial technique for dementia-related missing incidents. Importantly, we found no hotspots in the analysed region, indicating that the distribution of missing incidents is similar. This is an important null result as it highlights that missing incidents are not bound to specific locations but are an endemic symptom in dementia and therefore, part of the disease process [3]. The flipside is that for the police and search \& rescue services, it is not advisable to focus resources for dementiarelated missing incidents in certain regions. Rather, widespread information, training and support is required in response to the prevalent nature of the problem.

Despite not finding any spatial hotspots for MPWD, our second set of spatial results suggests that the increased presence of outdoor landmarks is an environmental risk factor contributing to patients getting lost, regardless of location. To our knowledge, this is the first time the effect of outdoor landmark presence on dementia patients getting lost has been explored in the real world. Previous studies have only investigated the role of landmarks in virtual environments (in lab settings) for patients [30,31], reporting impaired landmark recognition abilities in the patients. The exact mechanisms underlying why patients may be getting lost in real world environments with an increased landmark presence is at present unclear. A-priori we predicted that less landmarks would lead to more missing incidents, but our results showed the opposite effect. We know that landmarks play a key role in spatial navigation, 
functioning as building blocks for cognitive maps used predominantly in allocentric (i.e. map-based) navigation and in enabling us to orient ourselves to the surrounding environment for egocentric (i.e. body-based) navigation [32]. It was previously reported that when landmarks were increased in a virtual environment, healthy participants spent longer looking at these landmarks, which subsequently correlated with increased navigation duration [33]. This considered, one could speculate that the MPWD spent increased amounts of time fixating on the landmarks surrounding the location (that they were reported missing from). Moreover, with Alzheimer's disease patients being widely reported to be impaired in all aspects of spatial navigation [3], particularly landmark recognition [30,31], they might have been unable to utilise these outdoor landmarks effectively for navigation, contributing to their disorientation. Furthermore, it is possible that areas with less landmark density have more distinct landmarks whilst those with higher landmark density often contain objects/locations that repeat regularly (eg. franchise supermarkets, street lamps, bus stops, etc.). Considering this, it can be speculated that in the landmark dense areas, it is likely that the similarity of the repeating objects/locations to one another could have prevented them from being understandable landmarks for the MPWD, thereby challenging their navigation abilities in these areas.

With very little still being known about what real world factors underlie dementia-related missing incidents, our results provide novel evidence for increased presence of landmarks being a potential environmental risk factor for these occurrences. From a clinical perspective, our results suggest that patients living or navigating in regions dense with landmarks may be at high risk for getting lost, which may in turn inform caregivers to focus and implement safeguarding strategies to prevent this group from getting lost. Beyond the clinic our results have potential implications in urban/rural village planning, especially with regards to informing the placement as well as distribution of landmarks so as to make these regions 
more navigation friendly for dementia patients. This would indeed be an invaluable contribution to the development of dementia friendly communities.

Despite these exciting findings, there are some limitations to our study. The police data of MPWD was completely anonymised and hence did not allow to investigate further diagnosis or disease staging information for the MPWD. Clearly, investigating the effect of different dementia types and stages on missing incident patterns is important and needs to be addressed in future studies. In addition, the data did not contain any information about whether any of the community-dwelling MPWD lived alone or with a carer/spouse, hence not allowing further investigation into the influence of having a carer/spouse on missing incidents rates. The reported locations in the police data are the last known location of MPWD when they went missing but might not be the actual locations patients went missing from. Similarly, for landmark density it is not possible for us to know whether the patients might have actually noticed or used these landmarks whilst navigating. Lastly, it must be mentioned that since only missing incidents that were reported to the police were used in the study, the sample size utilised in this study may not be representative of the actual occurrences of MPWD in the region, likely underestimating the true extent of the problem. Still, despite these limitations which are common for retrospective analyses with given data, our results provide an important stepping stone towards more informed prospective studies using more refined geospatial analytical techniques.

Taken together, our results replicate and extend previous demographic findings for dementiarelated missing incidents. More importantly, we show that geospatial analytical techniques provide an exciting opportunity to determine systematic real-world factors that may contribute to dementia patients getting lost. In turn, these findings will inform future prospective studies and missing incidents guidelines, which are urgently needed to provide better safeguarding for dementia patients at risk of getting lost. 


\section{Acknowledgements}

This work was supported by the Earle \& Stuart Charitable Trust and the Faculty of Medicine and Health Sciences, University of East Anglia (Grant number R205319). We would like to thank Pippa Hinds and Mark Barnes from the Norfolk Constabulary Team for providing us with the missing patients with dementia case records. We would also like to thank Dr. Ed Manley and Prof. Andrew Lovett for the invaluable discussions during the data analysis.

\section{Conflict of Interest}

The authors have no conflict of interest to report. 


\section{References}

[1] Pengas G, Patterson K, Arnold RJ, Bird CM, Burgess N, Nestor PJ (2010) Lost and found: Bespoke memory testing for Alzheimer's disease and semantic dementia. $J$. Alzheimer's Dis. 21, 1347-1365.

[2] Tu S, Wong S, Hodges JR, Irish M, Piguet O, Hornberger M (2015) Lost in spatial translation - A novel tool to objectively assess spatial disorientation in Alzheimer's disease and frontotemporal dementia. Cortex 67, 83-94.

[3] Coughlan G, Laczó J, Hort J, Minihane AM, Hornberger M (2018) Spatial navigation deficits — overlooked cognitive marker for preclinical Alzheimer disease? Nat. Rev. Neurol. 14, 496-506.

[4] Rowe MA, Vandeveer SS, Greenblum CA, List CN, Fernandez RM, Mixson NE, Ahn HC (2011) Persons with dementia missing in the community: Is it wandering or something unique? BMC Geriatr. 11,

[5] National Policing Improvement Agency (2011) Alzheimer's Safe Return Project, , London.

[6] Alzheimer's Association. (2011) 2011 Alzheimer's disease facts and figures. Alzheimers Dement. 7, 208-44.

[7] Mcshane R, Gedling K, Keane J, Fairburn C, Jacoby R, Hope T (1998) Getting lost: a longitudinal study of a behavioural symptom. Int. Psychogeriatrics 10, 253-260.

[8] Rowe M, Houston A, Molinari V, Bulat T, Bowen M, Spring H, Mutolo S, McKenzie B (2015) The Concept of Missing Incidents in Persons with Dementia. Healthcare 3, $1121-1132$

[9] Bowen ME, McKenzie B, Steis M, Rowe M (2011) Prevalence of and antecedents to 
dementia-related missing incidents in the community. Dement. Geriatr. Cogn. Disord. 31, 406-412.

[10] Rowe MA, Bennett V (2003) A look at deaths occurring in persons with dementia lost in the community. Am. J. Alzheimers. Dis. Other Demen. 18, 343-348.

[11] Kwok TCY, Yuen KSL, Ho FKY, Chan WM (2010) Getting lost in the community: A phone survey on the community-dwelling demented people in Hong Kong. Int. J. Geriatr. Psychiatry 25, 427-432.

[12] Gallagher D, Ni Mhaolain A, Crosby L, Ryan D, Lacey L, Coen RF, Walsh C, Coakley D, Walsh JB, Cunningham C, Lawlor BA (2011) Determinants of the desire to institutionalize in Alzheimer's caregivers. Am. J. Alzheimers. Dis. Other Demen. 26, $205-211$.

[13] Greene KS, Pakes F (2014) The cost of missing person investigations: Implications for current debates.

[14] Robertson C, Nelson TA (2014) An Overview of Spatial Analysis of Emerging Infectious Diseases. Prof. Geogr. 66, 579-588.

[15] Bibby P (2013) The 2011 Rural-Urban Classification For Small Area Geographies: A User Guide and Frequently Asked Questions (v1.0).

[16] R Core Team (2017) R: A language and environment for statistical computing.

[17] Environmental Systems Research Institute (2015) ArcGIS 10.3.1.

[18] Census Geography.

https://www.ons.gov.uk/methodology/geography/ukgeographies/censusgeography\#sup er-output-area-soa 
[19] Lower Layer Super Output Areas (December 2011) Full Extent Boundaries in England and Wales, Last updated 2016, Accessed on 2016.

[20] Cliff AD, Ord JK (1973) Spatial autocorrelation, Pion, London.

[21] Anselin L. (1995) Local indicators of spatial association - LISA. Geogr. Anal. 27, 93115.

[22] Dabbs J., Chang EL, Strong R., Milun R (1998) Spatial ability, navigation strategy, and geographic knowledge among men and women. Evol. Hum. Behav. 19, 89-98.

[23] Grön G, Wunderlich AP, Spitzer M, Tomczak R, Riepe MW (2000) Brain activation during human navigation: Gender-different neural networks as substrate of performance. Nat. Neurosci. 3, 404-408.

[24] Rowe MA, Glover JC (2001) Antecedents, descriptions and consequences of wandering in cognitively-impaired adults and the Safe Return (SR) program. Am. J. Alzheimers. Dis. Other Demen. 16, 344-352.

[25] White EB, Montgomery P (2015) Dementia, walking outdoors and getting lost: Incidence, risk factors and consequences from dementia-related police missing-person reports. Aging Ment. Heal. 19, 224-230.

[26] Prince M, Knapp M, Guerchet M, McCrone P, Prina M, Comas-Herrera A, Wittenberg R, Adelaja B, Hu B, King D, Rehill A, Salimkumar D (2014) Dementia UK: Update (Second edition).

[27] Pai MC, Lee CC (2016) The incidence and recurrence of getting lost in communitydwelling people with Alzheimer's disease: A two and a half-year follow-up. PLoS One 11,

[28] Bagheri N, Wangdi K, Cherbuin N, Anstey KJ (2018) General Practice Clinical Data 
Help Identify Dementia Hotspots: A Novel Geospatial Analysis Approach. $J$. Alzheimer's Dis. 61, 125-134.

[29] Almeida MC da S, Gomes C de MS, Nascimento LFC (2014) Spatial distribution of deaths due to Alzheimer's disease in the state of São Paulo, Brazil. Sao Paulo Med. J. 132, 199-204.

[30] Allison SL, Fagan AM, Morris JC, Head D (2016) Spatial navigation in preclinical Alzheimer's disease. J. Alzheimer's Dis 52, 77-90.

[31] Zakzanis KK, Quintin G, Graham SJ, Mraz R (2009) Age and dementia related differences in spatial navigation within an immersive virtual environment. Med. Sci. Monit. 15, CR140-R150.

[32] O’Malley M, Innes A, Wiener JM (2017) Decreasing spatial disorientation in carehome settings: How psychology can guide the development of dementia friendly design guidelines. Dementia 16, 315-328.

[33] Andersen NE, Dahmani L, Konishi K, Bohbot V. (2012) Eye tracking, strategies, and sex differences in virtual navigation. Neurobiol. Learn. Mem. 97, 81-89. 


\section{Tables}

Table 1: Demographics of the Missing Dementia Patients

\begin{tabular}{|c|c|c|c|c|}
\hline & Total & Males & Females & Significance \\
\hline Cases & 210 & 114 & 96 & - \\
\hline Age (Median) & 81 & 80.5 & 81 & $\mathrm{p}=0.768$ \\
\hline \multicolumn{5}{|l|}{ Season Lost } \\
\hline Summer & 51 & 22 & 29 & \\
\hline Autumn & 52 & 29 & 23 & $p=0.317$ \\
\hline Winter & 52 & 31 & 21 & \\
\hline Spring & 55 & 32 & 23 & \\
\hline \multicolumn{5}{|l|}{ Setting Missing From } \\
\hline Domestic Residence & 134 & 63 & 71 & \\
\hline Care Facility & 52 & 36 & 16 & $\mathrm{p}=0.013 * 1$ \\
\hline Public Place & 23 & 15 & 8 & \\
\hline \multicolumn{5}{|l|}{ Locality Missing From } \\
\hline Urban & 134 & 75 & 59 & \\
\hline Rural & 73 & 37 & 36 & $\mathrm{p}=0.537$ \\
\hline Unspecified & 3 & 2 & 1 & \\
\hline $\begin{array}{l}\text { Distance Travelled } \\
\text { (Median; Meters) }\end{array}$ & 2000 & 1850 & 2000 & $\mathrm{p}=0.986$ \\
\hline $\begin{array}{c}\text { Time Missing } \\
\text { (Median; Minutes) }\end{array}$ & 55.5 & 74 & 43 & $\mathrm{p}=0.007 * * 2$ \\
\hline Missing Multiple Times & 52 & 37 & 15 & $\mathrm{p}=0.005^{* *}$ \\
\hline Sustained Harm & 10 & 5 & 5 & $\mathrm{p}=0.780$ \\
\hline
\end{tabular}

$1 *=\mathrm{p}<0.05$

$2 * *=\mathrm{p}<0.01$ 


\section{Figures}

i)

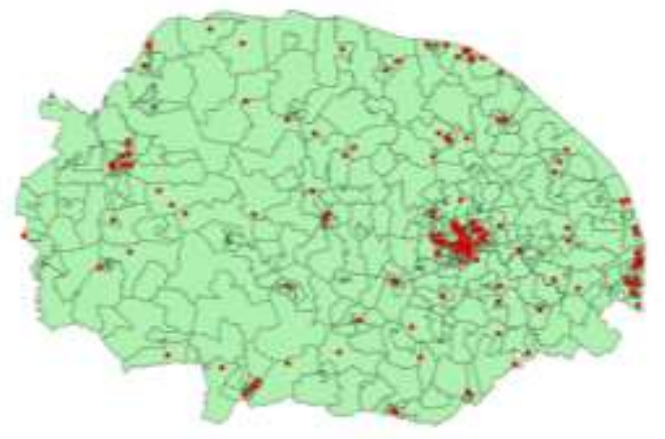

ii)

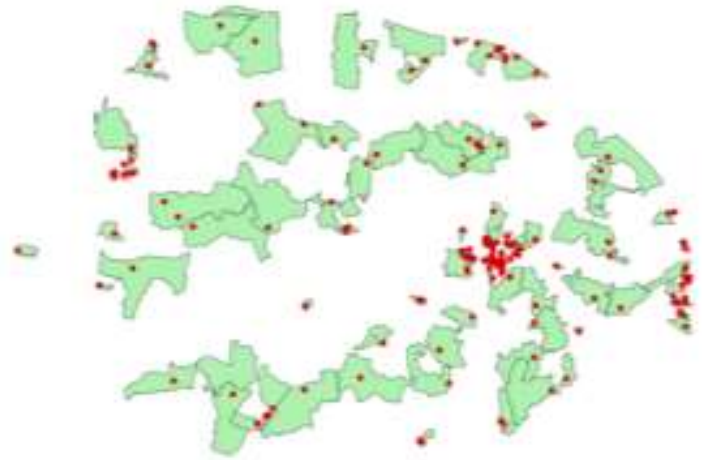

iii)

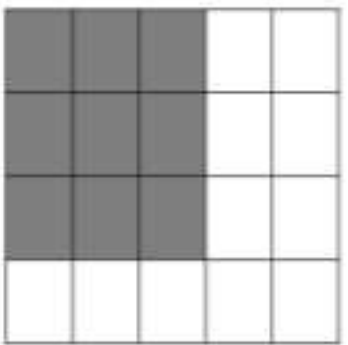

A

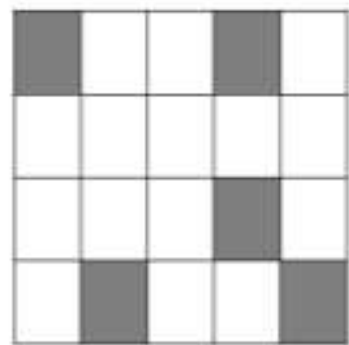

B

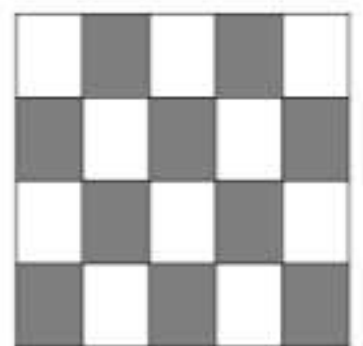

C

Figure 1(i): Locations MPWD went missing from plotted on a map of Norfolk county, subdivided into its LSOAs. (ii) Map of Norfolk county LSOAs after removing units with no MPWD. This map was used in the global spatial autocorrelation analysis. (iii) A: Positive Autocorrelation (maximum value +1 ). This suggests that the region of analysis is composed of LSOAs with similar MPWD values appearing near each other (i.e., spatial clusters). B: Zero Autocorrelation (0). This suggests that the region of analysis is composed of LSOAs exhibiting a completely random spatial pattern of MPWD values (i.e., no spatial clusters). C: Negative Autocorrelation (maximum value -1). This suggests that the region of analysis is composed of LSOAs with dissimilar MPWD values appearing near each other. 


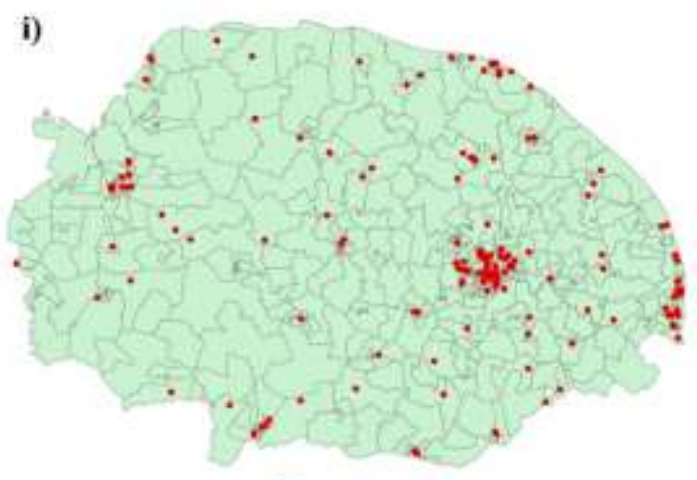

iii)
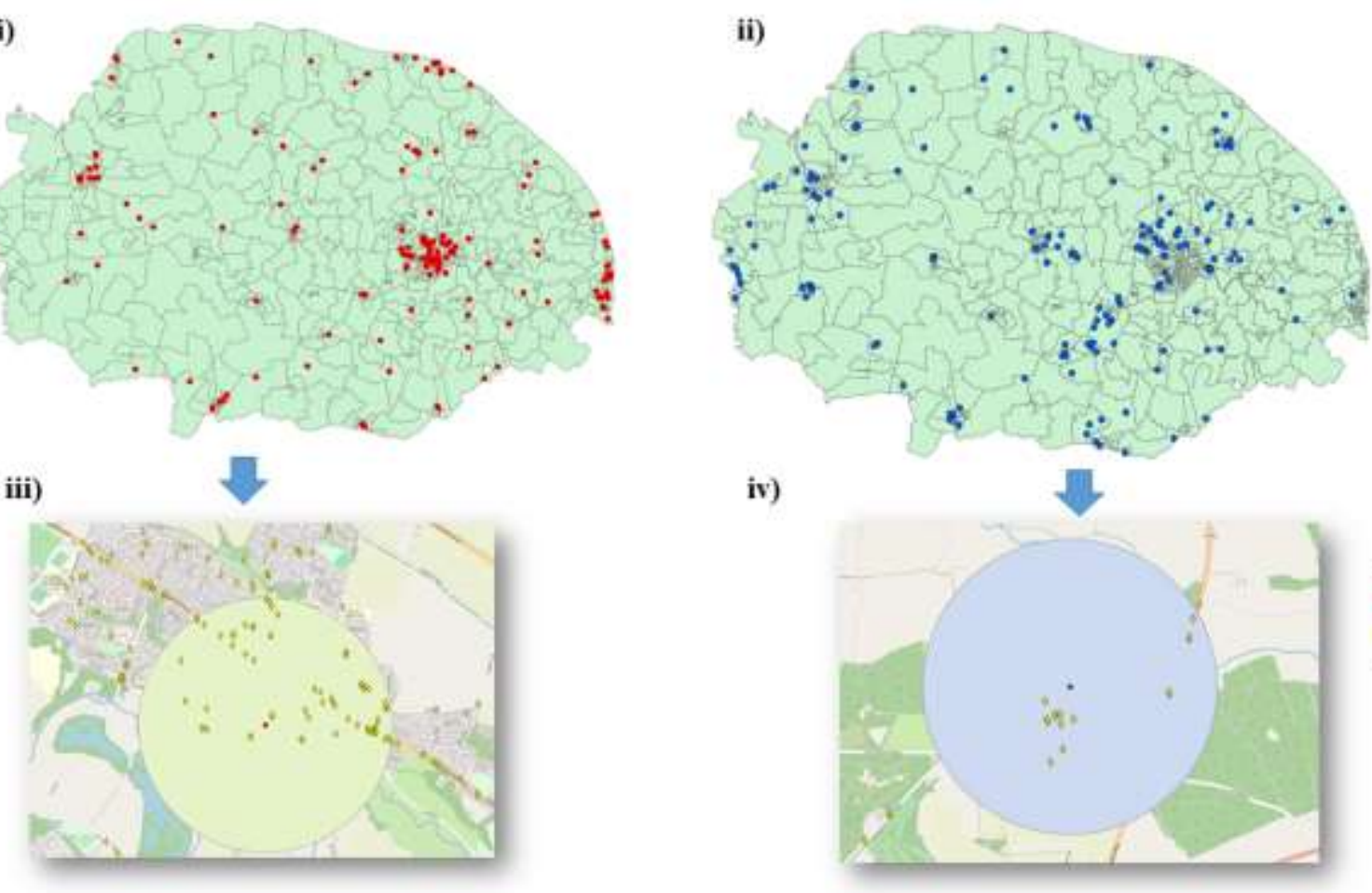

iv)

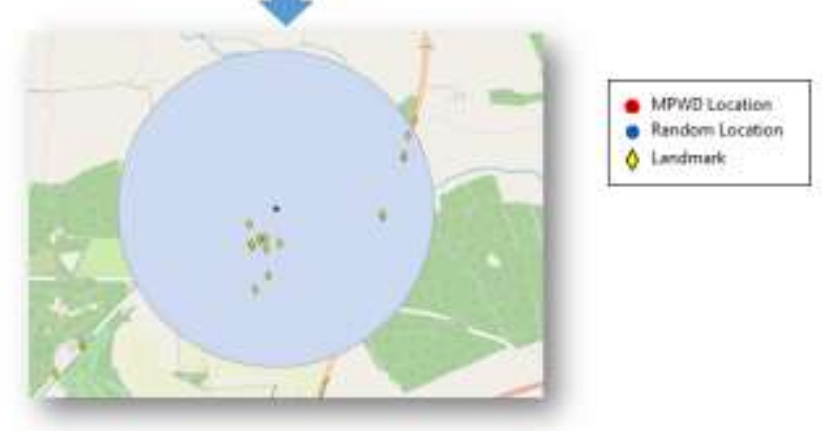

Figure 2(i): Locations MPWD went missing from in Norfolk. (ii): Set of random control locations in Norfolk generated using an in-built algorithm in ArcGIS. (iii): Landmarks falling within a 1 kilometre radius buffer zone of a single MPWD location (residential land). (iv): Landmarks falling within a 1 kilometre radius buffer zone of a single random location (residential land). 
SUPPLEMENTARY TABLE 1: List of Landmark Categories and Tags

\begin{tabular}{|c|c|c|}
\hline Category & Sub-Category & Tag \\
\hline \multirow{6}{*}{ Amenity and Leisure } & Food and Drink & $\begin{array}{l}\text { Bakery, Bar, Biergarten, Café, Fast Food, Green } \\
\text { Grocer, Pub, Restaurant, Supermarket }\end{array}$ \\
\hline & Leisure & $\begin{array}{l}\text { Arts Centre, Bank, Cinema, Clothes Store, } \\
\text { Community Centre, Computer Store Convenience } \\
\text { Stores, Department Stores, Do-It-Yourself Stores, } \\
\text { Dog Park, Florist, Furniture Store, Gift Store, Garden } \\
\text { Centre, Jeweller, Kiosk, Leisure Centres, Library, } \\
\text { Mobile Phone Store, Newsagent, Nightclub, Outdoor } \\
\text { Shop, Playground, Post Office, Other clubs and } \\
\text { centres, Service Centre, Shoe Store, Shopping Mall, } \\
\text { Stationery Store, Social Facility, Sports Centre, } \\
\text { Stadium, Studio, Swimming Centres, Theatre Toy } \\
\text { Store, Town Hall, Travel Agency, Video Store } \\
\text { Village Hall }\end{array}$ \\
\hline & Religious & $\begin{array}{l}\text { Church, Hindu Temple, Synagogue, Mosque, Sikh } \\
\text { Temple }\end{array}$ \\
\hline & Health and Beauty & $\begin{array}{l}\text { Beauty Shop, Chemist, Dentist, Doctors, Hairdresser, } \\
\text { Hospital, Laundry, Nursing Home, Optician, } \\
\text { Pharmacy, Veterinary }\end{array}$ \\
\hline & Education & Kindergarten, Nursery, School, University \\
\hline & Other & Graveyard, Prison \\
\hline \multirow[t]{3}{*}{ Tourism } & Attractions & $\begin{array}{l}\text { Local Attractions, Castle, Monument, Museum, } \\
\text { Parks, Theme Parks, Viewpoints, Zoo }\end{array}$ \\
\hline & Accommodation & $\begin{array}{l}\text { Guesthouse, Hostel, Hotel, Other Overnight } \\
\text { Accommodation, Motel }\end{array}$ \\
\hline & Information Points & Tourist Information Points, Visitors Centres \\
\hline Traffic and Transport & Transport Services & $\begin{array}{l}\text { Bus station, Bus Stop, Car Dealership, Car Rental, } \\
\text { Car Sharing, Car Wash, Crossing, Fire Station, Ferry } \\
\text { Terminal, Fuel Station, Marina, Parking Lots } \\
\text { (outdoor, multi-storey, underground), Bicycle } \\
\text { Parking, Police Station, Railway Platform, Railway } \\
\text { Halt, Railway Station, Other Transport Services, Taxi } \\
\text { Stand }\end{array}$ \\
\hline
\end{tabular}




\begin{tabular}{cl}
\hline Road Signs & Mini Roundabout, Stop, Traffic Signals \\
\hline Historic & Archaeological Sites, Memorials, Ruins \\
\hline Urban and Rural & \\
Furniture & Artwork, Arch, Art Space, ATM Machines, Aviary, \\
& Bandstand, Barn, Belfry, Bench, Bunker, Canopy, \\
& Control Tower, Communications Tower, Cowshed, \\
& Dove Cote, Drainage Pump, Gatehouse, Glasshouse, \\
& Greenhouse, Fountain, Lighthouse, Hut, Hangar, \\
& Kennels, Lych Gate, Marquee, Mill, Pagoda, \\
& Pavilion, Power Station, Pump House, Pumping \\
& Station, Observation Tower, Post Box, Recycling \\
& Containers, Silo, Stable, Storage (containers, tank), \\
& Street Lamp, Telephone Box, Toilet, Tower, Vending \\
& (machine, parking) Waste Basket, Water Tower, \\
& Water Well, Warehouse, Wayside Cross, Windmill, \\
& Wind Pump \\
& \\
\hline
\end{tabular}

